\title{
Regulation of hole transport layer for perovskite quantum dot light-emitting diodes
}

\author{
Ronghong Zheng ${ }^{1}$, Dong Huang ${ }^{1}$, Dongyang Shen ${ }^{1}$, Chengzhao Luo ${ }^{1}$ and Yu Chen ${ }^{1,2, *}$ \\ ${ }^{1}$ School of Optoelectronic Science and Engineering, Soochow University, Suzhou 215006, P. R. China \\ ${ }^{2}$ National University of Singapore Suzhou Research Institute Dushu Lake Science and Education Innovation District Suzhou 215123, P. \\ R. China
}

\begin{abstract}
Perovskite quantum dots have been widely used in light-emitting diodes (LEDs) because of their adjustable color, high quantum yield and easy solution processing. Furthermore, matching energy levels of device plays a profound role in the resultant LEDs. In this study, a polymeric material, namely poly[(9,9dioctylfluorenyl-2,7-diyl)-co-(4,4'-(N-(pbutylphenyl))diphenylamine)] (TFB), is introduced between the quantum dot emission layer and the hole injection layer PEDOT:PSS, which not only prevents the fluorescence quenching caused by the direct contact between the perovskite layer and the hole injection layer, but also reduces hole injection barrier, both being beneficial to the device performance. The optimal thickness of TFB has been obtained by adjusting the rotational speed and precursor solution concentration during spin coating. The optimized quantum dots LED has a switching on voltage of about $2.2 \mathrm{~V}$, a maximum brightness of $4300 \mathrm{~cd} / \mathrm{m}^{2}$, a maximum external quantum efficiency of $0.15 \%$, and a maximum current density of 0.54 cd/A.
\end{abstract}

\section{Introduction}

Owing to their tunable direct bandgap, high optical absorption coefficient, long carrier diffusion length and high defect tolerance, perovskites have been widely attempted in various optoelectronic applications, such as solar cells, light-emitting diodes, lasers and optical detectors. ${ }^{[1-7]}$ Peroveskite quantum dots have been widely reported as emissive materials in LEDs because of their high quantum yield, good monochromaticity (FWHM 20nm), adjustable color and other advantages. At present, the highest EQEs of three primary RGB colors have exceeded $20 \%,{ }^{[8]}$ for red and green and $10 \%$ for bule $^{[9]}$. Compared with OLED and inorganic quantum dot LED, perovskite quantum dots (QDs) possess additional advantages of wide color gamut, simple synthesis method, easily adjustable band gap, low cost and low environmental toxicity.

Generally, to obtain a high-performance LED, besides the optimization of the emissive perovskite materials, it is of highly importance to improve the device struture, including the selection of materials for each layer, the matching of energy levels, the wettability, film forming ability and carrier mobility of each layer. LED devices have been developed from simple sandwich structure to a more widely adopted multilayer structure, including anode, hole transport layer (HTL), emitting layer (EML), electron transport layer (ETL) and cathode. Owing to the fact that perovskite QDs were directly spin-coated on top of HTL during device fabrication, the selection and parameter of HTL greatly influence the overall performance of the
LEDs. Therefore, if the QDs were directly combined with the HTL material with large energy level difference and inappropiate thickness, fluorescence quenching and low injection efficiency may be resulted.

In this study, Poly[(9,9-dioctylfluorenyl-2,7-diyl)-co(4,4'-(N-(pbutylphenyl))diphenylamine)](TFB) was introduced as the hole transport layer, the thickness of which would have a profound influence on the device performance. By changing the rotational speed and precursor solution concentration of spin coating, the optimal thickness of $40 \mathrm{~nm}$ was obtained. This TFB layer not only reduced the fluorescence quenching, but also optimized the energy level matching and improved the hole injection efficiency. Finally, a quantum dot LED with a turn-on voltage of about $2.2 \mathrm{~V}$, a maximum brightness of $4300 \mathrm{~cd} / \mathrm{m}^{2}$, a maximum external quantum efficiency of $0.15 \%$ and a maximum current density of $0.54 \mathrm{~cd} / \mathrm{A}$ was obtained.

\section{Materials and Methods}

\subsection{Materials}

Poly(3,4-ethylenedioxythiophene):polystyrene sulfonate (PEDOT:PSS) was purchased from Heraeus Clevios. Poly[(9,9-dioctylfluorenyl-2,7-diyl)-co-(4,4'-(N-

(pbutylphenyl))diphenylamine)] (TFB) was purchased from American Dye Source Inc. Poly(methyl methacrylate) (PMMA) (average $\mathrm{M}_{\mathrm{w}} \approx 250000$ ) were purchased from Sigma-Aldrich. Al was purchased from Alfa Aesar. For the

\footnotetext{
* Corresponding author: chenyu_ny@suda.edu.cn
} 
synthesis of $\mathrm{ZnO}$ nanoparticles, a $0.5 \mathrm{M}$ solution of zinc acetate in dimethyl sulfoxide and $30 \mathrm{~mL}$ of tetramethylammonium hydroxide in ethanol were mixed and stirred in ambient air for 1 hour, then washed and dispersed in ethanol. Perovskite quantum dots, $\mathrm{FA}: \mathrm{CsPbBr}$, were synthesized by the triple-ligand surface engineering according to Song, J., et al. ${ }^{[10]}$ The solvents used in the experiment were purchased from Sigma and used directly.

\subsection{Methods}

Before the device was fabricated, the indium tin oxide (ITO) /glass substrate (15 $\Omega / \mathrm{sq}$ ) was ultrasonically treated with glass cleaning solution, deionized water, acetone and isopropanol for $15 \mathrm{~min}$ in sequence, and then dried with nitrogen flow. After treatment with ultraviolet (UV)-ozone plasma for 15 minutes, PEDOT:PSS was spin-coated onto the ITO substrate $(8000 \mathrm{rpm} / \mathrm{min}, 30 \mathrm{~s})$ and baked at $150{ }^{\circ} \mathrm{C}$ for 15 min under ambient conditions. Then the rest of the process took place in a glove box. Preparation of TFB chlorobenzene solution was carried out by spin-coating on the surface PEDOT:PSS and annealed at $150{ }^{\circ} \mathrm{C}$ for $30 \mathrm{~min}$. Then the perovskite quantum dots were spin-coated at $2000 \mathrm{rpm}$ for $2 \mathrm{~min}$ and baked at $60{ }^{\circ} \mathrm{C}$ for $2 \mathrm{~min}$. Next, a layer of PMMA (the thickness is about $10 \mathrm{~nm}$ ) was spin-coated and annealed at $60{ }^{\circ} \mathrm{C}, 15 \mathrm{~min}$. Subsequently, $\mathrm{ZnO}$ was spin-coated followed by annealing at $60{ }^{\circ} \mathrm{C}$ for $30 \mathrm{~min}$. Finally, $\mathrm{Al}$ were continuously deposited by thermal evaporation under high vacuum.

\section{Results and Discussion}

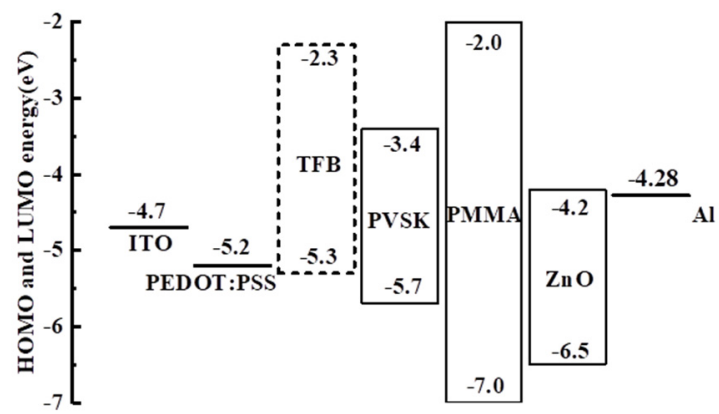

Figure 1. Energy level structure diagram of perovskite quantum dot light-emittting diodes

The

device

structure

of ITO/PEDOT:PSS/TFB/PVSK/PMMA/ZnO/Al was used to assemble perovskite quantum dot led (PQLED). PQLED is a device that converts the injected electrons and holes into photons through radiation recombination in quantum dots. If the density of injected electron is much larger than that of hole, and most of charge carriers fail to combine into effective electron hole pairs, resulting in low luminous efficiency. In order to balance the hole and electron concentrations at both ends of the quantum dot layer, improve the radiative recombination rate, and improve the luminous efficiency of the device, a hole transport layer and an electron barrier layer have been seperatedly added adjacent to the quantum dot layer. The electron barrier layer consisting of PMMA ${ }^{[14]}$ was aimed to reduce the free electron concentration, while the hole transport layer composed of $\mathrm{TFB}$ to facilitate hole injection. The energy level structure is shown in Figure 1. Before adding TFB, the barrier difference between the HOMO energy level of perovskite quantum dots and PEDOT:PSS was $0.5 \mathrm{eV}$. After adding TFB HTL, the injection loss of holes should be reduced. Besides, the carrier mobility of TFB is $1 \times 10^{-3} \mathrm{~cm}^{2} /(\mathrm{v} \cdot \mathrm{s})^{[11]}$, which is more favorable for hole transport compared with PVK (2.5 x $\left.10^{-6} \mathrm{~cm}^{2} /(\mathrm{v} \cdot \mathrm{s})\right)^{[12]}$ and Poly-TPD (1 $\mathrm{x}$ 10$\left.{ }^{4} \mathrm{~cm}^{2} /(\mathrm{v} \cdot \mathrm{s})\right)^{[13]}$. The LUMO energy level of TFB is $-2.3 \mathrm{eV}$, which could effectively block electron from entering HTL, thus avoiding recombination light emission in the TFB layer and effectively confining electron in perovskite layer. (a)

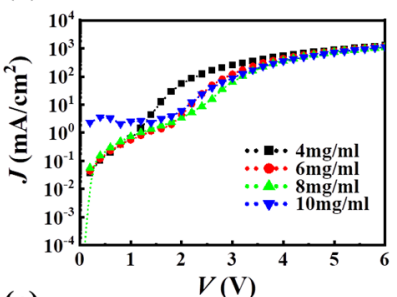

(c)

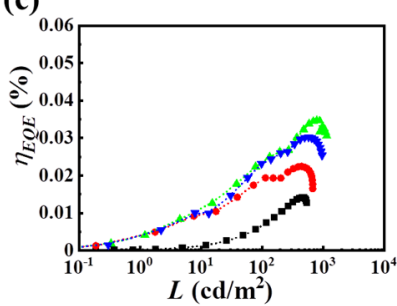

(b)

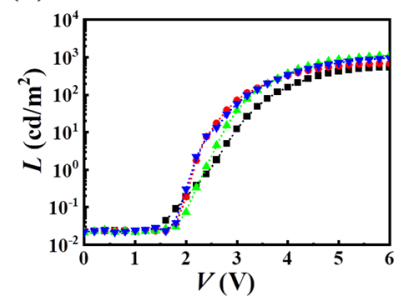

(d)

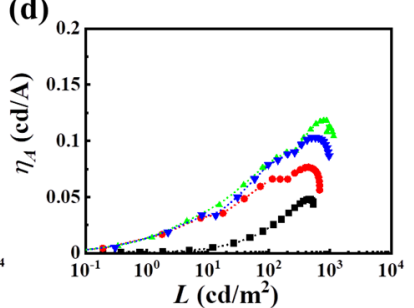

Figure 2. Device performances by changing TFB precusor concentration. (a) Current density-voltage characteristics, (b) luminance-voltage characteristics, (c) EQE and (d) current efficiency versus voltage of perovskite quantum dot lightemitting diodes.

The precursor solution concentration of TFB was firstly regulated. The chlorobenzene solutions of $4 \mathrm{mg} / \mathrm{ml}$, $6 \mathrm{mg} / \mathrm{ml}, 8 \mathrm{mg} / \mathrm{ml}$ and $10 \mathrm{mg} / \mathrm{ml}$ TFB were prepared, respectively, followed by spin-coating on top of PEDOT:PSS in a glove box. The performance of the manufactured devices are shown in Figure 2. The tested parameters include current density $\left(\mathrm{J}, \mathrm{mA} / \mathrm{cm}^{2}\right)$, luminance $\left(\mathrm{L}, \mathrm{cd} / \mathrm{m}^{2}\right)$, external quantum efficiency EQE and current efficiency $(\mathrm{CE}, \mathrm{cd} / \mathrm{A})$. The current density $(\mathrm{J}$, $\mathrm{mA} / \mathrm{cm}^{2}$ ) is an indication of the leakage current of the device after the voltage is applied. At low voltage, the smaller the current density, the less leakage current. Figure 2(a) shows the current density at different TFB precursor solution concentrations. Two curves corresponding to the TFB solutions with concentration of 6 and $8 \mathrm{mg} / \mathrm{ml}$ showed less leakage current compared to the rest. Figure 2(b) is the luminance and voltage curve. The turn-on voltages (corresponding voltage when the brightness is $1 \mathrm{~cd} / \mathrm{m}^{2}$ ) of different TFB concentrations are $2.5 \mathrm{~V}, 2.3 \mathrm{~V}$, $2.2 \mathrm{~V}$, and $2.1 \mathrm{~V}$, respectively. Figure $2(\mathrm{c})$ and $(\mathrm{d})$ are the curves of external quantum efficiency and current efficiency versus brightness. Among them, the maximum 
value of EQE and current efficiency were about $0.035 \%$, $0.13 \mathrm{~cd} / \mathrm{A}$, respectively, both of which corresponded to the TFB concentration of $8 \mathrm{mg} / \mathrm{ml}$. The test results are recorded in Table 1.

Table 1. Various parameters of perovskite quantum dot lightemitting diodes with different TFB precursor concentrations

\begin{tabular}{|c|c|c|c|c|}
\hline $\begin{array}{c}\text { TFB } \\
\text { Concentration } \\
(\mathrm{mg} / \mathrm{ml})\end{array}$ & 4 & 6 & 8 & 10 \\
\hline $\mathrm{L}_{\max }\left(\mathrm{cd} / \mathrm{m}^{2}\right)$ & 538 & 674 & 1141 & 958 \\
\hline $\begin{array}{c}\text { Turn-on } \\
\text { Voltage }(\mathrm{V})\end{array}$ & 2.5 & 2.2 & 2.3 & 2.1 \\
\hline $\mathrm{EQE}_{\max }(\%)$ & 0.01 & 0.02 & 0.035 & 0.03 \\
\hline $\mathrm{CE}_{\max }(\mathrm{cd} / \mathrm{A})$ & 0.05 & 0.07 & 0.13 & 0.1 \\
\hline
\end{tabular}

Subsequently, the rotational speed of TFB has been adjusted by fixing the TFB precursor concentration at $8 \mathrm{mg} / \mathrm{ml}$. Specifically, rotational speed of 3000rpm, 3500rpm, 4000rpm, 4500rpm, 5000rpm, 5500rpm, 6000rpm, and 6500rpm have been applied. The corresponding device performances are shown in Figure 3. (a)
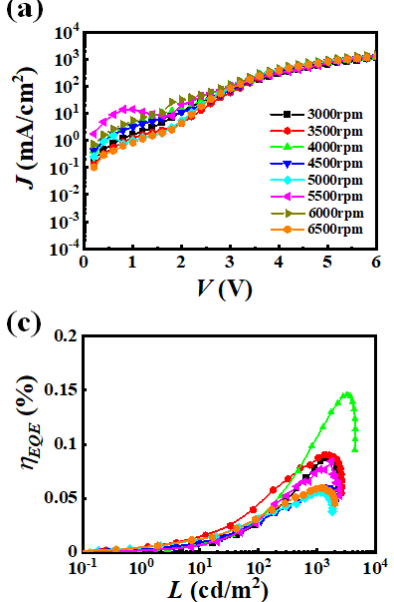

(b)

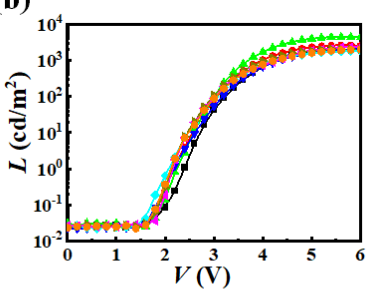

(d)

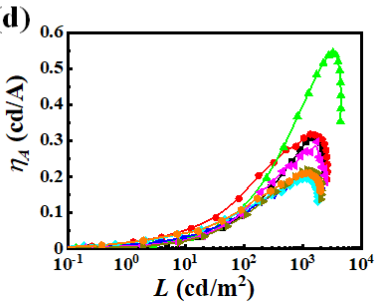

Figure 3. LED performances after optimization of TFB spin coating rotational speed. (a) J-V characteristics, (b) L-V characteristics, (c) EQE and (d) Current efficiency versus voltage of PQLEDs.

Figure 3 (a) shows the current density versus voltage curves at different rotational speeds of TFB precursor. It is found by comparison that when the speed was $4000 \mathrm{rpm}$, the leakage current was small. Figure 3(b) is the luminance and voltage curve of the perovskite quantum dots LED. When the brightness was $1 \mathrm{~cd} / \mathrm{m}^{2}$, the turn-on voltage corresponding to 4000rpm was 2.2 V. From Figure 3(c) and $3(\mathrm{~d})$, the maximum EQE was about $0.15 \%$, and the maximum $\mathrm{CE}$ was about $0.54 \mathrm{~cd} / \mathrm{A}$, both corresponding to the rotational speed of $4000 \mathrm{rpm}$. According to the control of concentration and rotational speed, it is found that the perovskite quantum dots LED demonstrated the best deivce performances when the TFB concentration was $8 \mathrm{mg} / \mathrm{ml}$ and the rotational speed was $4000 \mathrm{rpm}$. The test results are recorded in Table 2 .
Table 2. Various parameters of perovskite quantum dot lightemitting diodes with different rotational speed of TFB precursor solutions.

\begin{tabular}{|c|c|c|c|c|}
\hline Speed & $\begin{array}{c}\mathrm{L}_{\max } \\
\left(\mathrm{cd} / \mathrm{m}^{2}\right)\end{array}$ & $\begin{array}{c}\text { Turn-on } \\
\text { Voltage } \\
(\mathrm{V})\end{array}$ & $\begin{array}{c}\mathrm{EQE}_{\max } \\
(\%)\end{array}$ & $\begin{array}{c}\mathrm{CE}_{\max } \\
(\mathrm{cd} / \mathrm{A})\end{array}$ \\
\hline $3000 \mathrm{rpm}$ & 2034 & 2.4 & 0.08 & 0.31 \\
\hline $3500 \mathrm{rpm}$ & 2594 & 2.2 & 0.09 & 0.32 \\
\hline 4000rpm & 4380 & 2.2 & 0.15 & 0.54 \\
\hline 4500rpm & 2354 & 2.2 & 0.06 & 0.20 \\
\hline 5000rpm & 2022 & 2.1 & 0.05 & 0.19 \\
\hline 5500rpm & 2415 & 2.2 & 0.08 & 0.30 \\
\hline 6000rpm & 2540 & 2.2 & 0.06 & 0.22 \\
\hline 6500rpm & 2354 & 2.1 & 0.06 & 0.21 \\
\hline
\end{tabular}

Figure 4(a) shows the picture under natural light just after spin coating of TFB with the optimal combination of precursor concentration and rotational speed. It can be seen from the figure that the film formation is uniform throughout the substraute. Figure 4(b) shows the luminescence diagram of the perovskite quantum dot device after power-on. From the figure, it can be observed that the luminous area emits uniformly without fluorescence blinking. Figure 4(c) shows the crosssectional view of the fabricated PQLED. From top to bottom, layers corresponding to ITO, PEDOT: PSS, TFB, PVSK, PMMA, ZnO, and Al can be clearly observed. As shown in the figure, the thicknesses of PEDOT:PSS and PMMA were relatively thin, being about $20 \mathrm{~nm}$ and $10 \mathrm{~nm}$ respectively. The thickness of TFB was about $40 \mathrm{~nm}$, which is the optimal thickness after adjustment. (a)

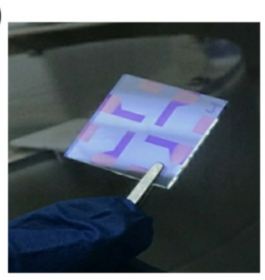

(c)

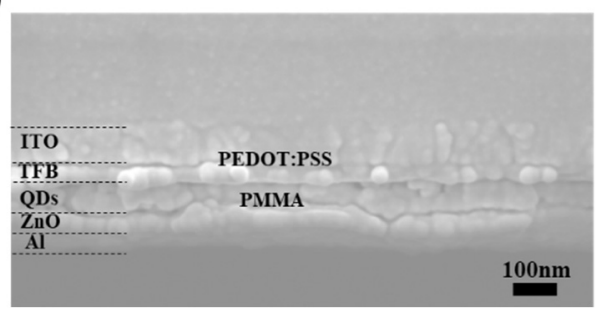

Figure 4. (a) The picture under natural light after spinning TFB. (b) The lit up picture after device fabrication. (c) A crosssectional view of the overall device. 


\section{Conclusion}

In summary, we regulated the hole transport layer TFB between the perovskite layer and the hole injection layer PEDOT:PSS to optimize the energy level matching of the overall device. Besdies, this addtional TFB layer is beneficial to the effective hole injection and able to prevent the fluorescence quenching caused by the direct contact between the perovskite luminescent layer and the hole injection layer. On the other hand, it blocks electrons and avoids recombination of charge carrier in the TFB layer. By adjusting the rotational speed and concentration of the TFB precursor solution, the optimal thickness has been obtained, so that holes passed through effectively, finally recombined with electrons in the perovskite layer to realize effective green light emission. The optimal result of the device is a turn-on voltage of $2.2 \mathrm{~V}$, a maximum brightness of $4300 \mathrm{~cd} / \mathrm{m}^{2}$, a maximum external quantum efficiency of $0.15 \%$, and a maximum current density of $0.54 \mathrm{~cd} / \mathrm{A}$. Such results will help the development of perovskite LEDs and lays the foundation for their potential commercial applications.

\section{Acknowledgments}

This work was supported by the Natural Science Foundation of Jiangsu Province of China (Grant Nos. BK20190098, the Natural Science Foundation of the Jiangsu Higher Education Institutions of China (19KJA550001), Key Lab of Modern Optical Technologies of Education Ministry of China, Key Lab of Advanced Optical Manufacturing Technologies of Jiangsu Province, Priority Academic Program Development (PAPD) of Jiangsu Higher Education Institutions, and Jiangsu Shuangchuang Plan.

\section{References}

1. Zhou, H., Chen, Q., Li, G., Luo, S., Song, T.-B., Duan, H.-S., Hong, Z., You, J., Liu, Y. and Yang, Y. (2014) Interface Engineering of Highly Efficient Perovskite Solar Cells. Science, 345, 542-546.

2. Xing, G., Mathews, N., Sun, S., Lim, S.S., Lam, Y.M., Gratzel, M., Mhaisalkar, S. and Sum, T.C. (2013) Long-Range Balanced Electron- and Hole-Transport Lengths in Organic-Inorganic CH3NH3PbI3. Science, 342, 344-347.

3. N.-G. Park, Mater. Today, 2014, 18, 65-72.

4. J. Kim, S.-H. Lee, J. H. Lee, and K.-H. Hong, J. Phys. Chem. Lett. 5, 1312 (2014).

5. T. Baikie, Y. Fang, J.M. Kadro, M. Schreyer, F. Wei, S.G. Mhaisalkar, M. Graetzel, T.J. White, J. Mater. Chem. A1, 5628 (2013).

6. Y. Miyazawa, M. Ikegami, H.-W. Chen, T. Ohshima, M. Imaizumi, K. Hirose and T. Miyasaka, iScience, 2018, 2, 148-155.

7. Guo, H., Zhang, H., Shen, C., Zhang, D., Liu, S., Wu, Y. and Zhu, W.-H. (2021), A Coplanar $\pi$-Extended Quinoxaline Based Hole - Transporting Material
Enabling over $21 \%$ Efficiency for Dopant - Free Perovskite Solar Cells. Angew. Chem. Int. Ed.

8. Shen, H., Gao, Q., Zhang, Y. et al. Visible quantum dot light-emitting diodes with simultaneous high brightness and efficiency. Nature Photon 13, 192197 (2019).

9. Karlsson, M., Yi, Z., Reichert, S. et al. Mixed halide perovskites for spectrally stable and high-efficiency blue light-emitting diodes. Nat Commun 12,361 (2021).

10. Song, J., Li, J., Xu, L., Li, J., Zhang, F., Han, B., Shan, Q., Zeng, H., Adv. Mater. 2018, 30, 1800764.

11. Tang, P.; Xie, L.; Xiong, X.; Wei, C.; Zhao, W.; Chen, M.; Zhuang, J.; Su, W.; Cui, Z. Realizing 22.3\% EQE and 7-Fold Lifetime Enhancement in QLEDs Via Blending Polymer TFB and Cross-Linkable Small Molecules for a Solvent-Resistant Hole Transport Layer. ACS Appl. Mater. Interfaces 2020, 12, 13087- 13095.

12. Thesen, M. W. et al. Hole-transporting host-polymer series consisting of triphenylamine basic structures for phosphorescent polymer light-emitting diodes. $J$. Polym. Sci. A 48, 3417-3430 (2010).

13. Dai, X., Zhang, Z., Jin, Y. et al. Solution-processed, high-performance light-emitting diodes based on quantum dots. Nature 515, 96-99 (2014). 